Abstracta Iranica Abstracta Iranica

Revue bibliographique pour le domaine irano-aryen

Volume 40-41 | 2019

Comptes rendus des publications de 2017-2018

\title{
Alireza Anisi (ed.). Adle Nāmeh. Studies in Memory of Chahriyar Adle
}

Sandra Aube

\section{OpenEdition}

1 Journals

\section{Édition électronique}

URL : http://journals.openedition.org/abstractairanica/52158

DOI : 10.4000/abstractairanica.52158

ISBN : 1961-960X

ISSN : 1961-960X

Éditeur :

CNRS (UMR 7528 Mondes iraniens et indiens), Éditions de l'IFRI

Référence électronique

Sandra Aube, "Alireza Anisi (ed.). Adle Nāmeh. Studies in Memory of Chahriyar Adle », Abstracta Iranica [En ligne], Volume 40-41 | 2019, document 7, mis en ligne le 30 décembre 2019, consulté le 16 avril 2021. URL : http://journals.openedition.org/abstractairanica/52158 ; DOI : https://doi.org/10.4000/ abstractairanica. 52158

Ce document a été généré automatiquement le 16 avril 2021.

Tous droits réservés 


\title{
Alireza Anisi (ed.). Adle Nāmeh. Studies in Memory of Chahriyar Adle
}

\author{
Sandra Aube
}

\section{RÉFÉRENCE}

Alireza Anisi (ed.). Adle Nāmeh. Studies in Memory of Chahriyar Adle. Tehran: Research Institute for Cultural Heritage and Tourism, 2018. ISBN: 978-600-8977-36-0 (anglaispersan, 594 p.)

1 Ce volume est un hommage à Chahriyar Adle (1942-2015), paru à titre posthume. L'ouvrage rassemble 22 articles scientifiques signés par 29 chercheurs : des collègues et amis d'Adle, et d'éminents spécialistes des arts et de l'archéologie du monde iranien. Toutes ces contributions reflètent remarquablement les différents champs de recherche abordés par Adle au cours de sa carrière. Directeur de recherche au CNRS, historien de l'art de formation, Adle a notamment encouragé l'étude de terrains et de ressources matérielles originales, principalement sur l'Iran médiéval et moderne. Il a ainsi abordé des domaines et des méthodologies variées, allant des arts du livre jusqu'aux archives cinématographiques, en passant par des prospections archéologiques et des études architecturales, principalement en Iran. L'éclectisme des contributions offertes dans ce livre traduisent le caractère pluriforme de sa recherche et l'étendue des problématiques abordées.

2 L'ouvrage est organisé en deux parties indépendantes: l'une en persan et l'autre en anglais (contenant deux contributions en français). Chaque section est préfacée par l'éditeur scientifique, Alireza Anisi.

3 La section en persan ouvre avec un hommage de Seyyed Muhammad Beheshti, associée à la liste complète des publications de Chahriyar Adle (jusqu'à une liste de ses enseignements et de communications données). Cette introduction est ensuite suivie de 12 articles abordant des thèmes et des périodes des plus variés :

4 - Alireza Qasim Khan, « Diver of Images » [en persan] (p. 49-58), 
5 - Sa'ied Qutbizadeh, «Chahriyar Adle's Achievement in the History of Iranian Cinema » [en persan] (p. 59-63),

6 - Alireza Anisi, «Imamzadeh Ja 'far at Damghan, an Architectural Study » [en persan] (p. 65-94),

7 - Assadullah Judaki Azizi et Seyyed Rasul Mousavi Haji, «Re-examination of Central Kushk at Lashgargah, Bust, Afghanistan » [en persan] (p. 95-114),

8 - Maryam Rezaiepour et Hamidreza Jeyhani, «Re-examination of Safavid Herat According to the Decree of Shah Tahmasp I » [en persan] (p. 115-134),

9 - Mehdi Rahbar, «Providing Light and Heat in the Parthian Buildings at Khurhe » [en persan] (p. 135-159),

10 - Muhammad Ibrahim Zar'ie, «Evolution of the Mosque of Bayazid at Bastam Complex » [en persan] (p. 161-188),

11 - Ali Sadraie, « Landscape Archaeology of the House of God » [en persan] (p. 189-203),

12 - Hasan Fazli Nashli et Seyyed Taha Hashimi, «Social and Cultural Evolutions from Anthropology and Archaeology Points of View » (p. 205-240),

13 - Muhammad Murtizaie et Zahra Poursh'abaniyan, « Historical Geography of Khurasan in the Medieval Islamic Texts » (p. 241-256),

14 - Ahmad Mirzakuchak Khushnevis, «Triumphs of Majestic » (p. 257-268),

15 - Farhad Nazari, « Behavior towards the Past Relics » (p. 269-299).

16 La section en anglais commence par un hommage à Ch. Adle signé de Yann Richard et Nader Nasiri-Moghaddam. Suivent 10 articles scientifiques :

17 - Sheila Blair, «The Archeaology of a Manuscript » (p. 15-34),

18 - Abbas Daneshvari, «Allusions to $9^{\text {th }}$ and $10^{\text {th }}$ Century Khurāsān Gardens on Their Contemporary Ceramics » (p. 35-52),

19 - Robert Hillenbrand, «Building Sites in Medieval Persian Painting » (p. 53-74),

20 - Lorenz Korn, «Ribat-i Mahi (Khurasan-i Razavi, Iran): Evidence of a Seljuq Inscription » (p. 75-97),

21 - Assadullah Souren Melikian-Chirvani, «Qajar Revivalims: Dreams of a Lost Past» (p. 99-132),

22 - Milo C. Beach, «Muraqqa'-e Gulshan: The Artists of the Marginal Figures » (p. 133-166),

23 - Nader Nasiri Moghaddam, «Persian Documents in the National Archives (Torre do Tombo) of Portugal and their importance for the history of Persian Gulf in the $16^{\text {th }}-17^{\text {th }}$ centuries »(p. 167-194),

24 - Susan Stronge et Behnaz Atighi Moghaddam, "An Unrecorded Polier Muraqqa (c. 1785). New Insights into British-Hindustani Cultural Interaction » (p. 195-228),

25 - Willem Floor et Gholam Vatandoust, «Jūwak-kārī, A Neglected Persian Art Form or Qajar Paintings on Wood» (p. 229-261),

26 - Yves Porter, « Revêtements émaillés des premiers Moghols à Delhi » (p. 263-299).

27 On pourra sans doute regretter l'absence de relation ou de dialogue entre ces deux parties; on conçoit cependant que des impératifs purement éditoriaux aient probablement dicté cette stricte séparation entre la recherche iranienne et occidentale. 
Le système double de pagination rend parfois malaisée la recherche au sein de l'ouvrage -Notons que nous avons renvoyé ici à la pagination persane, qui semble avoir prévalu dans l'organisation de l'ouvrage. Des résumés bilingues, de même qu'une organisation peut-être plus thématique, auraient sans doute été appréciés. Mais ces quelques remarques n'atténuent en rien l'intérêt d'un ouvrage qui rassemble un nombre conséquent de recherches originales sur les arts et l'archéologie de l'Iran. C'est à l'évidence un bel hommage rendu à Chahryar Adle.

\section{AUTEURS}

SANDRA AUBE

CNRS, CeRMI 